\title{
Shape-shifting polyhedral droplets
}

\author{
Pierre A. Haas,,${ }^{1,}$ Diana Cholakova, ${ }^{2}$ Nikolai Denkov, ${ }^{2}$ Raymond E. Goldstein $\odot,{ }^{1}$ and Stoyan K. Smoukov ${ }^{3, \dagger}$ \\ ${ }^{1}$ Department of Applied Mathematics and Theoretical Physics, Centre for Mathematical Sciences, University of Cambridge, \\ Wilberforce Road, Cambridge CB3 OWA, United Kingdom \\ ${ }^{2}$ Department of Chemical and Pharmaceutical Engineering, Faculty of Chemistry and Pharmacy, University of Sofia, 1164 Sofia, Bulgaria \\ ${ }^{3}$ School of Engineering and Materials Science, Queen Mary, University of London, Mile End Road, London E1 4NS, United Kingdom
}

(Received 19 February 2019; published 16 September 2019)

\begin{abstract}
Cooled oil emulsion droplets in aqueous surfactant solution have been observed to flatten into a remarkable host of polygonal shapes with straight edges and sharp corners, but different driving mechanisms-(i) a partial phase transition of the liquid bulk oil into a plastic rotator phase near the droplet interface and (ii) buckling of the interfacially frozen surfactant monolayer enabled by a drastic lowering of surface tension-have been proposed. Here, combining experiment and theory, we analyze the initial stages of the evolution of these "shape-shifting" droplets, during which a polyhedral droplet flattens into a polygonal platelet under cooling and gravity. Using reflected-light microscopy, we reveal how icosahedral droplets evolve through an intermediate octahedral stage to flatten into hexagonal platelets. This behavior is reproduced by a theoretical model of the phase transition mechanism, but the buckling mechanism can only reproduce the flattening if the deformations are driven by buoyancy. This requires surface tension to decrease by several orders of magnitude during cooling and yields bending modulus estimates orders of magnitude below experimental values. The analysis thus shows that the phase transition mechanism underlies the observed "shape-shifting" phenomena.
\end{abstract}

DOI: 10.1103/PhysRevResearch.1.023017

\section{INTRODUCTION}

The culmination of the geometric preoccupations of Ancient Greece was doubtless the classification of the five platonic solids [1]. It is topology, however, that dictates that one of their number, the icosahedron, should abound in nature, among the shapes of virus capsids and other biological structures [2]: Euler's formula implies the formation of at least 12 topological defects in a hexagonal lattice on the surface of a spherical vesicle. By virtue of their elastic properties [3], these defects repel each other [4] to arrange at the vertices of a platonic icosahedron.

These same topological considerations play their part in the phenomenon of "shape-shifting" droplets reported by Denkov et al. [5]: Micron-sized oil droplets in aqueous surfactant solution flatten, upon slow cooling, into a plethora of polygonal shapes with straight edges and sharp vertices [Fig. 1(a)]. Although first revealed briefly over a decade ago [6,7], these phenomena generated a veritable flurry of largely experimental papers [5,8-21] only more recently. These studies revealed that the shape-shifting phenomena occur for a humongous range of surfactants and pure organic phases or mixtures thereof $[9,11]$, and showed how to harness these phenomena for efficient, controlled self-emulsification [10,12]. More

\footnotetext{
*P.A.Haas@ damtp.cam.ac.uk

${ }^{\dagger}$ Corresponding author: s.smoukov@qmul.ac.uk
}

Published by the American Physical Society under the terms of the Creative Commons Attribution 4.0 International license. Further distribution of this work must maintain attribution to the author(s) and the published article's title, journal citation, and DOI. recent studies demonstrated their manufacturing potential by synthesizing small polymeric particles $[13,19]$; scaled-up versions of these bottom-up approaches may enable massively parallel control over internally determined particle shape and particle uniformity that are currently only available in topdown approaches such as lithography techniques $[13,19]$.

Understanding the mechanisms driving these striking yet simple phenomena is therefore not only a beautiful research problem at the interface of chemistry, physics, and applied mathematics: it is a compulsory step for further studies of these manufacturing applications, without which their development is limited to trial-and-error approaches. Yet, in spite of the large number of experimental studies, these mechanisms remain debated. There is agreement that the initial deformations of the droplets are caused by freezing of the surfactant adsorption layer [5,8,16-18] and the ensuing topological frustration of the hexagonal packing of the surfactant molecules therein, with the formation of 12 defects arranged at the vertices of a regular icosahedron [16-18]. Two driving mechanisms for the subsequent deformations have, however, been proposed: (i) a partial phase transition of the bulk oil phase $[5,8,9,12]$, and (ii) elastic buckling of the frozen surfactant layer [16-18]. According to the first mechanism, as the droplets are cooled and the surfactant layer freezes, the formation of a plastic rotator phase $[22,23]$ becomes energetically favorable next to the droplet surface [Fig. 1(b)]. In this rotator phase, the oil molecules have long-range translational order. The rotator phase then arranges into a scaffold of plastic rods at the surface of the droplet supporting the faceted droplet structure [Fig. 1(c)]. We have shown in earlier theoretical work [15] that the rotator-phase mechanism can account for the sequence of polygonal shapes seen in experiments, the 
(a)

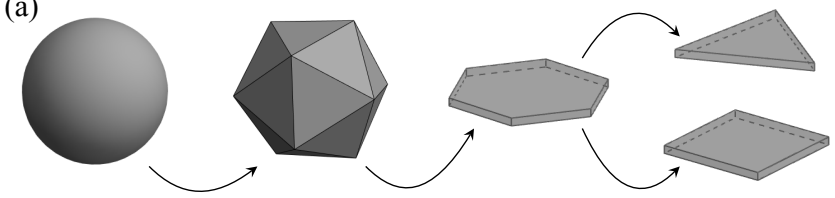

(b) ${ }_{\uparrow} \Delta G$

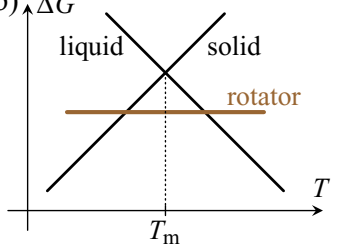

(c) mechanism (i) $\mid$ mechanism (ii)

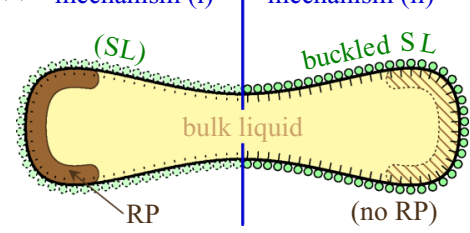

FIG. 1. Shape-shifting droplets. (a) Main stages of the droplet shape evolution, following Refs. [5,9]: The initially spherical droplets become icosahedral due to the interplay of topology and elasticity. Subsequently, the droplets flatten into hexagonal platelets which then evolve into triangles or quadrilaterals. (b) Energy diagram: In a range of temperatures close to the melting temperature $T_{\mathrm{m}}$, the formation of a rotator phase is energetically favored. (c) Mechanisms for the shape shifting of droplets, illustrated in the cross section of a flattened droplet: According to mechanism (i) (left), deformations are driven by a partial phase transition of the bulk liquid into a plastic rotator phase (RP) near the drop surface. According to mechanism (ii) (right), deformations are driven by buckling of the frozen surfactant adsorption layer (SL). See text for a further description of the mechanisms. The figure panels have been redrawn and modified from Ref. [15].

statistics of shape outcomes, and the observation that some droplets puncture before freezing [5]. According to the second mechanism, cooling the droplets reduces their surface tension to ultralow values, enabling elastic deformations to dominate over surface energy and hence causing the buckled droplet surface to deform [Fig. 1(c)]. The two mechanisms thus differ only in the respective origins of the contributions of the edges of the polyhedral droplets to their energy: the formation of a rotator phase at the edges by a phase transition of the bulk oil and surface elasticity of the buckled edges of the polyhedral droplet.

The early three-dimensional polyhedral stages of the droplet evolution, from an initial icosahedron down to a flattened hexagon [Fig. 1(a)], still need to be explored, however. They are the subject of this paper: Here, we analyze the flattening of an icosahedral droplet into a hexagonal platelet in detail, comparing experimental observations to predictions of mathematical models describing either mechanism to decide which mechanism underlies the observed phenomena.

In Sec. II of this paper, we thus reveal, using reflectedlight microscopy, how an icosahedral droplet flattens via an intermediate octahedral stage. We extend the model of the rotator-phase mechanism of Ref. [15] to three-dimensional polyhedral droplets, and we introduce a model of the elastic buckling mechanism in Sec. III. Additional physics arises in the extended rotator-phase model through a microscopic law, absent from the earlier analysis in Ref. [15], encoding the local effect of the dihedral angles of the polyhedral droplets on the phase transition. Through a linear stability analysis and numerical calculations in Sec. IV, we show that the rotatorphase mechanism can reproduce the observed flattening

dynamics if and only if such a microscopic law is included in the model. By contrast, the results in Sec. IV show that the elastic buckling mechanism can only reproduce the observed deformations if the process is driven by the interplay of elasticity and buoyancy. This requires surface tension to decrease by at least four orders of magnitude, yet the resulting estimate of the bending modulus of the droplets obtained in Sec. IV is three orders of magnitude below its experimental values. The analysis therefore suggests that it is the formation of a rotator phase rather than elastic buckling at ultralow surface tension that drives the shape-shifting processes observed so far $[5,8,9,11,18,19]$.

\section{EXPERIMENTAL FLATTENING DYNAMICS}

The flattening of the shape-shifting droplets under cooling and the stages of the droplet evolution intermediate between the initial spherical stage and the later flattened stages were observed using reflected-light microscopy to determine the three-dimensional shapes of the droplets at different stages of their evolution (Fig. 2). The experimental setup is described in Appendix A.

The droplets are initially spherical. Two well-defined types of images can be observed, depending on the position of the focal plane of the microscope: First, if the microscopy focus is on the top of the drop, just below the level of the upper wall of the glass capillary containing the emulsion, circular diffraction fringes (Newton rings) are seen [Fig. 2(a)]. These fringes emerge from the interference of the light reflected from the two surfaces of the aqueous film, formed between the wall of the glass capillary and the surface of the spherical oil droplet [Fig. 2(a)]. Second, if the microscope is focused on the equatorial plane of the droplet instead, a bright circle around the particle periphery is observed, due to light refraction and reflection at the drop surface [Fig. 3(a)].

Deformation of the droplets begins with the appearance of 12 vertices on the drop surface [Figs. 2(b) and 3(b)]. With the focal plane at the droplet equator, all 12 vertices can be observed simultaneously as three black dots (representing the three upper vertices next to the glass capillary), three white dots (representing the three vertices at the bottom of the droplet), and six bright spots at the drop periphery [Fig. 3(b)]. The latter correspond to three vertices just above and three vertices just below the equatorial plane of the droplet, which explains why they have a slightly different appearance in the microscopy images in Fig. 3(b). As a result, the spherical droplet distorts and it soon acquires an icosahedral shape; we note that this ideal shape transformation is, however, only observed in some of the systems under appropriate conditions such as slow cooling [5,9]. At the same time, the shape of the aqueous film between the glass capillary and the oil droplet becomes triangular, with rounded corners [Fig. 2(b)]. Upon further cooling, the drop continues to deform so that this aqueous film appears as an equilateral triangular shape with sharp corners [Fig. 2(c)].

As cooling continues, this triangular film increases its area significantly. At the same time, the cross section of the droplet equator also increases in size. Since the volume of the droplet is conserved, the droplet flattens in the perpendicular direction. Although the droplet resembles a hexagonal prism 

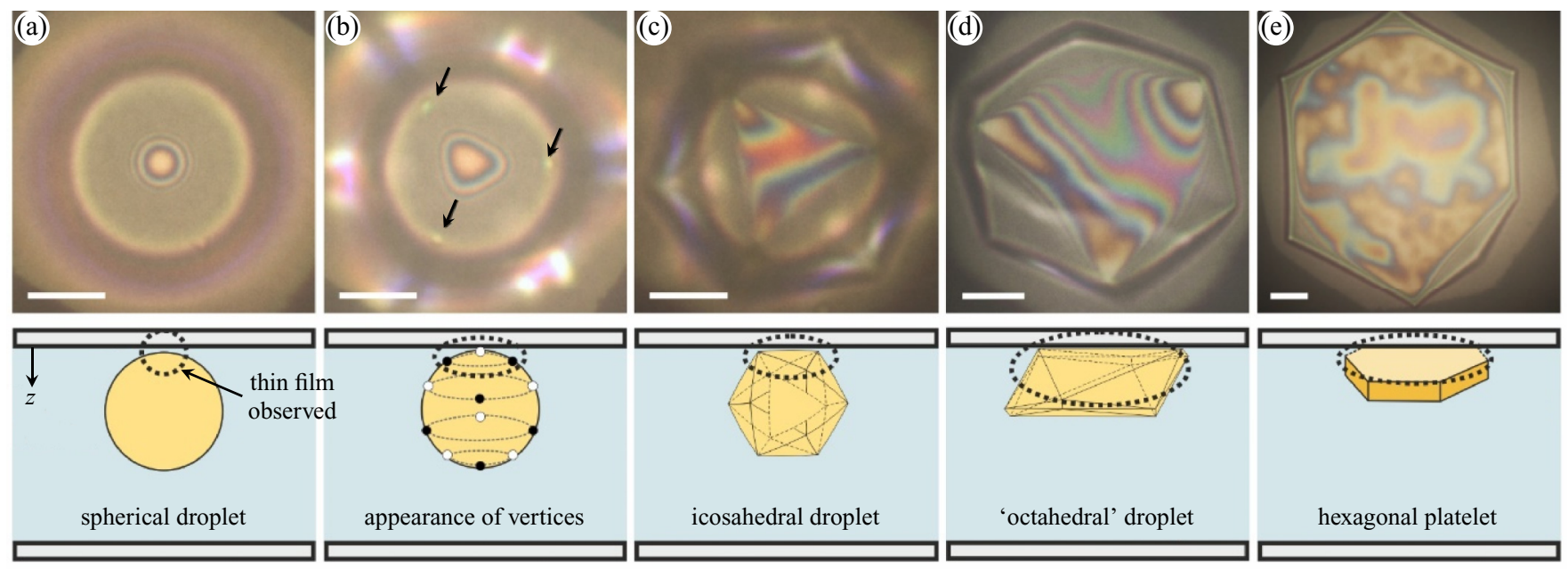

FIG. 2. Droplet shape evolution observed in reflected light. Top row: Microscopy images obtained upon cooling of a hexadecane emulsion droplet immersed in $1.5 \mathrm{wt}$ \% Tween 60 surfactant solution; scale bars: $10 \mu \mathrm{m}$. Bottom row: Sketch of side view of droplet deformations. Dotted circles and ellipses show the part of the drop observed in reflected-light experiments. (a) Before the drops start to deform, the aqueous film formed between the top of the drop and the wall of the glass capillary appears as circular Newton rings in reflected light. (b) The emulsion film deforms into a triangular shape when the drop begins to deform. Black arrows show three of the 12 vertices formed on the drop surface at this stage. (c) Upon further cooling, the triangular film expands until its corners engulf the three vertices at the periphery of the droplet that are situated above the equatorial plane of the icosahedral droplet. The three vertices forming the bottom surface of the drop appear as bright spots. (d) As the flattening process continues, pairs of vertices of the icosahedron come closer to each other: The droplet becomes octahedral. (e) As the droplet flattening completes, six pairs of vertices merge and the drop becomes a hexagonal platelet.

in transmitted light at this stage, the images in reflected light reveal that the three-dimensional drop shape is better represented as a distorted flattened icosahedron [Fig. 2(d)]. The flattening of the icosahedral droplet drives pairs of vertices closer to each other, so that the droplet assumes the shape of a flattened octahedron. Eventually, pairs of vertices can merge to form true hexagonal platelets [Fig. 2(e)], but the details of this final step depend on the system (Appendix A).

To understand these complex droplet shape deformations, we derive theoretical models corresponding to the two proposed mechanisms $[5,8,9,16-18]$ in the next section.
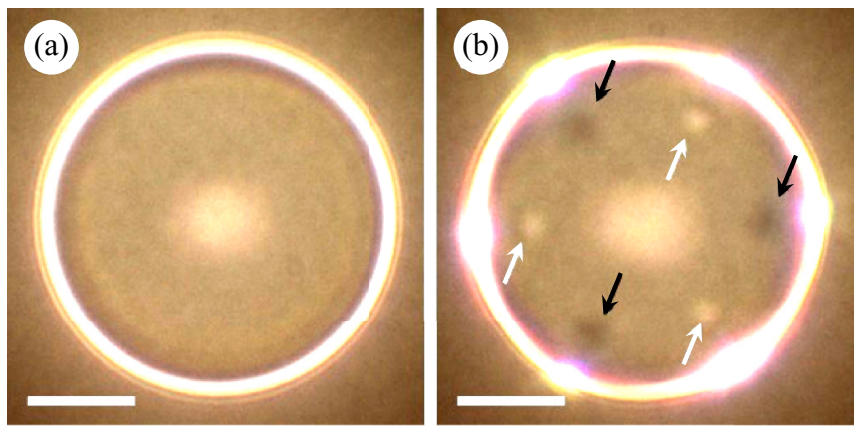

FIG. 3. Initial stages of the droplet shape deformations observed in reflected light with a focus on the equatorial plane of the droplet. (a) Before the droplet starts to deform, the droplet equator appears as a homogeneous bright circle. (b) As the drop begins to deform, 12 vertices are observed: Dark dots and bright spots (marked with black and white arrows) represent, respectively, the vertices at the top and bottom surfaces of the droplet. The remaining six vertices are located at the drop periphery, just above and below the equatorial plane of the droplet. Scale bars: $10 \mu \mathrm{m}$.

\section{MODEL}

On purely combinatorial grounds, the appearance of octahedral droplets as the vertices of the initial icosahedron merge during the flattening process is not surprising: Indeed, the octahedron is one of only two polyhedra with six vertices that can be obtained by edge contraction from an icosahedron, and the only one that does not require additional symmetry breaking (Appendix B). Static, entropic considerations of this ilk cannot, however, capture the dynamics of the problem: As in our previous theoretical description of the dynamics of flattened polygonal droplets [15], to describe the deformations of a polyhedron, we must specify (i) a (nondimensional) energy $\mathcal{E}$, and (ii) a kinetic law that relates energy gradients to deformations of the polyhedron.

In this paper, we model the polyhedral droplets as convex polyhedra of fixed volume $V$, with flat faces. Throughout the paper, we shall use $\mathscr{E}$ and $\mathscr{F}$ to denote, respectively, the set of edges and faces of such a polyhedral droplet.

\section{A. Droplet energy}

In this section, we show that the two mechanisms that have been proposed to explain the shape-shifting phenomena $[5,8,9,16-18]$ can be described by nondimensional energies of the form

$$
\mathcal{E}=\sum_{f \in \mathscr{F}}\|f\| \pm C \sum_{e \in \mathscr{E}}\|e\|^{a} \mathcal{F}(\delta(e)),
$$

wherein $\|f\|$ is the nondimensionalized area of face $f,\|e\|$ is the nondimensionalized length of edge $e$, and the dimensionless function $\mathcal{F}$ depends only on the dihedral angle $\delta(e)$ at edge $e$. The sign of the second term, the parameter $C$, the 
value of the exponent $a$, and the functional form of $\mathcal{F}$ depend on the details of the mechanism, as discussed below.

Throughout the paper, we shall use subscripts 1 to refer to the rotator-phase mechanism, and subscripts 2 to refer to the elastic buckling mechanism.

\section{Rotator-phase mechanism}

For the rotator-phase mechanism, as in our previous work [15], conservation of droplet volume implies $V=V_{\mathrm{r}}+V_{1}$, where $V_{\mathrm{r}}, V_{1}$ are the respective volumes of the rotator and liquid phases [24]. The energy $E_{1}$ of the droplet, with contributions from surface tension and from the rotator phase, is therefore

$$
E_{1}=\gamma S+\mu_{\mathrm{r}} V_{\mathrm{r}}+\mu_{1} V_{1}=\gamma S-\left(\mu_{1}-\mu_{\mathrm{r}}\right) V_{\mathrm{r}}+\mu_{1} V,
$$

wherein $\gamma$ is the surface tension, $S$ is the surface area, and $\mu_{\mathrm{r}}, \mu_{1}$ are the respective chemical potentials (per unit volume) of the rotator and liquid phases.

The rotator phase is assumed to form preferentially near the edges of the polyhedron, and we denote by $V_{\mathrm{r}}(e)$ the volume of rotator phase that forms near edge $e$. We approximate $V_{\mathrm{r}}(e)=A(e) \ell(e)$, where $\ell(e)$ denotes the length of edge $e$, and $A(e)$ is the cross-sectional area of the rotator phase (Fig. 4). This is where additional physics comes in compared to the two-dimensional, polygonal case: For a polyhedral droplet, $A(e)$ depends in general on the dihedral angle $\delta(e)$ at edge $e$ (Fig. 4), and we write $A(e)=A_{\mathrm{r}} F(\delta(e))$, where $A_{\mathrm{r}}$ is a characteristic cross-sectional area of the rotator phase and where $F$ is a dimensionless factor that we discuss further below.

We nondimensionalize Eq. (2) by scaling lengths with the radius $R \approx 10 \mu \mathrm{m}$ of the shape-shifting droplet [5]. On discarding the constant contribution $\mu_{1} V$ to Eq. (2), the nondimensional energy $\mathcal{E}_{1}=E_{1} / \gamma R^{2}$ is thus

$$
\mathcal{E}_{1}=\sum_{f \in \mathscr{F}}\|f\|-\alpha \sum_{e \in \mathscr{E}}\|e\| F(\delta(e)),
$$

which is of the form announced in Eq. (1). The model involves a single dimensionless number, the tension

$$
\alpha=\frac{A_{\mathrm{r}} \Delta \mu}{\gamma R},
$$

where $\Delta \mu=\mu_{1}-\mu_{\mathrm{r}}>0$ is the difference of the chemical potentials of the liquid and rotator phases, as in our previous work [15]. The parameter $\alpha$ thus compares the tendency to form a rotator phase to the stabilizing effect of surface tension. Since $\gamma$ and $\Delta \mu$ depend on temperature, so does $\alpha$;

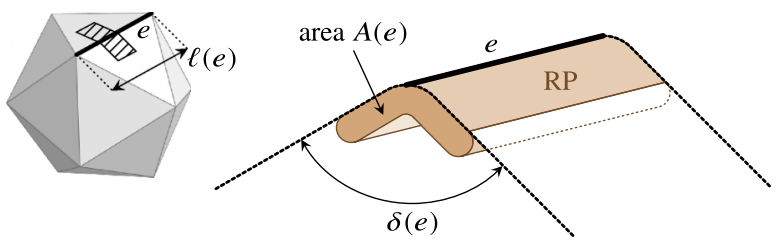

FIG. 4. Definition of geometric quantities used in the derivation of the rotator-phase model: Cross section of an edge $e$ of a polyhedral droplet (inset). The edge has length $\ell(e)$ (inset) and dihedral angle $\delta(e)$. The cross-sectional area of the rotator phase (RP) that has formed near $e$ is $A(e)$.

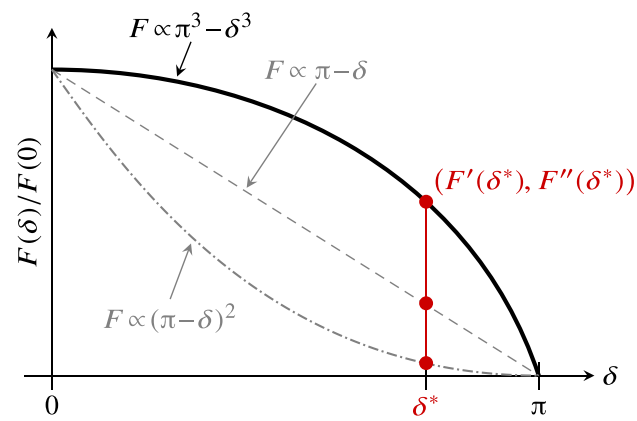

FIG. 5. Microscopic law. Plot of $F(\delta)$ against dihedral angle $\delta$, showing different functional forms satisfying conditions (5). Only the local behavior of $F$ near $\delta=\delta^{*} \approx 138.2^{\circ}$, the dihedral angle of a platonic icosahedron, affects the linear stability analysis in Sec. IV A, but the global behavior enters the numerical calculations in Sec. IV B, with droplet flattening only obtained for sufficiently concave choices of $F$.

previous estimates [15] suggest that $O(0.1) \lesssim \alpha \lesssim O(10)$ in experiments.

The function $F$ encodes the dependence of the formation of the rotator phase on the dihedral angle $\delta(e)$ at edge $e$. The dihedral angles and hence the values of $F$ are constant during the polygonal stages of the shape evolution, which is why this function did not arise in our previous work [15]. The detailed functional form of $F$ is set by the microscopic properties of the rotator phase $[9,23]$, but we expect $F$ to be a decreasing function of $\delta(e)$, with

$$
F(0) \geqslant F(\delta(e)) \geqslant F(\pi)=0,
$$

as shown in Fig. 5. Hence no rotator phase is formed when the two faces adjacent to edge $e$ are parallel to each other, while the tendency to form the rotator phase is maximal when the two faces have folded on top of each other, in agreement with the discussion in Refs. [9,23].

Deriving the functional form of $F$ is a wholly separate problem. This issue is rather akin, conceptually, to the analysis of a Landau theory of some system: There, one does not need to know how the coefficients of the truncated expansion defining the Landau theory relate to microscopic properties of the system to be able to study the phase transitions that arise in this system. This analogy will become perhaps clearest in the linear stability analysis of a platonic icosahedron in Sec. IV A, where only the local behavior of $F$ near its dihedral angle $\delta^{*} \approx 138.2^{\circ}$ matters (Fig. 5). More generally, however, the numerical calculations in Sec. IV B will enable us to constrain the functional form of $F$ more globally, beyond the conditions expressed by Eq. (5), even without further knowledge about the microscopic details that set $F$.

\section{Elastic buckling mechanism}

For the elastic buckling mechanism, the energy $E_{2}$ has contributions from surface tension and from surface elasticity,

$$
E_{2}=\gamma S+U,
$$

where, again, $\gamma$ is the surface tension and $S$ is the surface area. Here, we are interested in the regime where sharp elastic ridges form along the edges of the polyhedron. In this regime, 
corresponding to large values of the dimensionless Föppl-von Kármán number [25], the elastic energy of the ridges is much larger than the contributions to $U$ from defect elasticity [25]. These elastic ridges have been analyzed in Ref. [26], which thus yields the dimensional scaling $U \sim E h^{8 / 3} R^{1 / 3}$ [27], in which $E$ is the elastic modulus of the frozen surfactant adsorption layer, $h$ is its thickness, and $R \approx 10 \mu \mathrm{m}$ is again the typical radius of a shape-shifting droplet. Scaling lengths with $R$, the nondimensional energy $\mathcal{E}_{2}=E_{2} / \gamma R^{2}$ of the system is thus

$$
\mathcal{E}_{2}=\sum_{f \in \mathscr{F}}\|f\|+\beta \sum_{e \in \mathscr{E}}\|e\|^{1 / 3}[\pi-\delta(e)]^{7 / 3},
$$

which is, again, of the form announced in Eq. (1). The dependence of the second, elastic term in Eq. (7) on the nondimensionalized length $\|e\|$ of edge $e$ and its dihedral angle $\delta(e)$ follows from the scalings in Ref. [26]. Again, the energy depends on a single dimensionless coefficient,

$$
\beta \sim \frac{E h^{8 / 3}}{\gamma R^{5 / 3}},
$$

which thus expresses the relative magnitude of elasticity and surface tension; $\beta$ depends on temperature since $\gamma$ does.

In the above calculation, we have neglected the energetic contributions from the defects, and, in particular, from defectdefect interactions. As remarked above, the results of Ref. [25] show that this approximation is justified at least initially, but it must break down when defect pairs become close to each other at later stages of the flattening. For this reason, this model cannot be used to analyze the merging of vertices, i.e., the formation of a truly polygonal droplet. Similarly, the assumption inherent in the rotator-phase model, that the energetic contributions from the rotator phase swamp those associated with defect elasticity, must break down at these late stages.

Upon including an additional nondimensionalized buoyancy term $\mathcal{B}$, this energy becomes instead

$$
\mathcal{E}_{2}^{\prime}=\mathcal{E}_{2}+\text { Bo } \mathcal{B}
$$

which introduces an additional dimensionless number: The Bond number $\mathrm{Bo}=\Delta \rho g R^{2} / \gamma$ measures the relative magnitude of buoyancy and surface tension effects [29]. Here, $\Delta \rho \approx 250 \mathrm{~kg} / \mathrm{m}^{3}$ is the density difference between the water and oil phases [30], and $g \approx 9.81 \mathrm{~m} / \mathrm{s}^{2}$ is the acceleration due to gravity.

There is no consensus on the value of $\gamma$ at the temperature $T_{\mathrm{d}}$ at which the deformations are first observed [8,9,18], and the proponents of the elastic buckling mechanism indeed argue that the observed deformations are driven by ultralow or even transiently negative values of $\gamma$ [16-18]. Nevertheless, using the estimate $\gamma \approx 5 \mathrm{mN} / \mathrm{m}$ above $T_{\mathrm{d}}$ that the different experimental analyses $[8,9,18]$ agree on, we estimate $\mathrm{Bo} \approx 10^{-4}$, and conclude that $\gamma$ must decrease by at least four orders of magnitude during cooling for buoyancy effects to play a role.

Comparing Eqs. (3) and (7), we see that, as announced earlier, the two mechanisms only differ in the functional form of the contributions to the energy of the edges of the polyhedron. To understand the effect of this difference, it is useful to consider the platelet stages of the droplet evolution, which, for the rotator-phase mechanism, we have previously described by modeling the droplets as polygons [15]. For the rotator-phase mechanism, disproportionation of the side lengths of a polygonal droplet of fixed area is energetically favorable because of geometry: Among all polygons of a fixed number of vertices and fixed area, the regular one has the least perimeter [31]. By contrast, in the elastic buckling mechanism, disproportionation of the side lengths of a polygonal droplet follows from a convexity argument: Disproportionation is energetically favorable because the second term in Eq. (7) is a concave function of edge length [32].

\section{B. Kinetic law}

We relate energy gradients to deformations of the polyhedron by imposing a kinetic law. Standard surface-driven kinetic laws [33] relate energy variations to the (scalar) normal velocity of the surface (i.e., of the faces of the polyhedron). In our case, however, the motion is driven by the edges of the polyhedron rather than by its faces: In the first mechanism, rotator phase forms at the edges only, while in the second mechanism, the elastic energy is concentrated in the ridges at the edges of the polyhedron. We therefore impose a (nondimensional) edge-driven kinetic law,

$$
\sum_{e \in \mathscr{E}} \int_{e} \dot{r}_{\mathbf{n}} \cdot \delta \boldsymbol{r}_{\mathbf{n}} d \ell=-\delta \mathcal{E},
$$

wherein $\boldsymbol{r}_{\mathbf{n}}$ is the (vector) normal displacement of a point $\boldsymbol{r}$ on edge $e$ of the polyhedron and dots denote differentiation with respect to time. This is the three-dimensional analog of the two-dimensional kinetic law that we have used previously to describe the polygonal stages of the droplet evolution [15], and it expresses the requirement that the normal velocity of the edges (which is in this case, in three dimensions, a vector) be proportional to the energy gradient. Nevertheless, the equations corresponding to the more standard surface-driven kinetic law [33] can be derived by analogous reasoning, and we have checked that choosing this surface-driven kinetic law yields results in qualitative agreement with those presented below for the edge-driven kinetic law (10).

Consider the edge $e$ joining vertices $\boldsymbol{a}$ and $\boldsymbol{b}$, and let $\boldsymbol{t}$ denote the unit tangent parallel to it (Fig. 6). As the polyhedron deforms from its initial shape, described by some variables $\boldsymbol{x}$, to a new shape described by $\boldsymbol{x}+\delta \boldsymbol{x}$, the vertices $\boldsymbol{a}, \boldsymbol{b}$ are mapped to $\boldsymbol{a}^{\prime}, \boldsymbol{b}^{\prime}$, respectively. A Taylor expansion yields $\boldsymbol{a}^{\prime}=\boldsymbol{a}+\mathrm{A} \cdot \delta \boldsymbol{x}+\boldsymbol{O}\left(|\delta \boldsymbol{x}|^{2}\right)$ and $\boldsymbol{b}^{\prime}=\boldsymbol{b}+\mathrm{B} \cdot \delta \boldsymbol{x}+O\left(|\delta \boldsymbol{x}|^{2}\right)$, wherein $\mathrm{A}=\nabla_{x} \boldsymbol{a}, \quad \mathrm{B}=\nabla_{x} \boldsymbol{b}$ are the respective gradients of $\boldsymbol{a}, \boldsymbol{b}$ with respect to $\boldsymbol{x}$. Hence, assuming that the edges stretch uniformly, a point $\boldsymbol{r}=\boldsymbol{a}+(\boldsymbol{b}-\boldsymbol{a}) s$ on $e$, where $0 \leqslant s \leqslant 1$, is mapped to $\boldsymbol{r}^{\prime}=\boldsymbol{r}+\mathrm{R} \cdot \delta \boldsymbol{x}+O\left(|\delta \boldsymbol{x}|^{2}\right)$, with $\mathrm{R}=\mathrm{A}+(\mathrm{B}-\mathrm{A}) s$, as shown in Fig. 6. The $O\left(|\delta \boldsymbol{x}|^{2}\right)$ corrections can be neglected in what follows.

Let $\mathrm{P}=\mathrm{I}-\boldsymbol{t} \boldsymbol{t}$, where I the identity, denote projection onto the plane normal to $\boldsymbol{t}$ [34]. Then $\delta \boldsymbol{r}_{\mathbf{n}}=\mathrm{P} \cdot \delta \boldsymbol{r}=\mathrm{P} \cdot \mathrm{R} \cdot \delta \boldsymbol{x}$, as shown in Fig. 6, and so $\dot{\boldsymbol{r}}_{\mathbf{n}}=\mathrm{P} \cdot \mathrm{R} \cdot \dot{\boldsymbol{x}}$. By direct computation or Ref. [34], $\mathrm{P}^{2}=\mathrm{P}$, and so

$$
\int_{e} \dot{\boldsymbol{r}}_{\mathbf{n}} \cdot \delta \boldsymbol{r}_{\mathbf{n}} d \ell=\|e\| P_{i j} \delta x_{k} \dot{x}_{\ell} \int_{0}^{1} R_{i k} R_{j \ell} d s,
$$




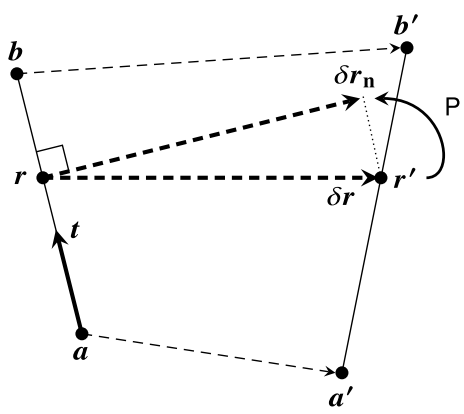

FIG. 6. Derivation of the kinetic law: Definition of the normal displacement. As the polyhedron deforms, the end points $\boldsymbol{a}, \boldsymbol{b}$ of an edge $e$ with unit tangent $\boldsymbol{t}$ move to $\boldsymbol{a}^{\prime}, \boldsymbol{b}^{\prime}$. A point $\boldsymbol{r}$ on $e$ is mapped to $\boldsymbol{r}^{\prime}=\boldsymbol{r}+\delta \boldsymbol{r}$; its normal displacement $\delta \boldsymbol{r}_{\mathbf{n}}$ is the projection, represented by the matrix $\mathrm{P}$, of $\delta \boldsymbol{r}$ onto the plane normal to $\boldsymbol{t}$.

where we have changed variables using $d \ell=\|e\| d s$. On letting $S=A+B$,

$$
\int_{0}^{1} R_{i k} R_{j \ell} d s=\frac{1}{6}\left(S_{i k} S_{j \ell}+A_{i k} A_{j \ell}+B_{i k} B_{j \ell}\right) .
$$

The factor $1 / 6$ in this equation can and will be scaled out by rescaling time (and hence velocities). Thence

$$
-\frac{\partial \mathcal{E}}{\partial x_{k}} \delta x_{k}=\dot{x}_{\ell} \delta x_{k} \sum_{e \in \mathscr{E}}\|e\| P_{i j}\left(S_{i k} S_{j \ell}+A_{i k} A_{j \ell}+B_{i k} B_{j \ell}\right),
$$

which leads to the overdamped evolution equation

$$
\dot{x}=-\mathrm{M}^{-1} \cdot \nabla_{x} \mathcal{E},
$$

wherein $M$ is the mobility matrix, defined by

$$
\mathrm{M}=\sum_{e \in \mathscr{E}}\|e\|\left(\mathrm{S}^{\top} \cdot \mathrm{P} \cdot \mathrm{S}+\mathrm{A}^{\top} \cdot \mathrm{P} \cdot \mathrm{A}+\mathrm{B}^{\top} \cdot \mathrm{P} \cdot \mathrm{B}\right) .
$$

\section{RESULTS}

\section{A. Linear stability analysis}

A necessary (albeit not sufficient) condition for droplet flattening is that the initial regular icosahedron be unstable to small perturbations. For the linear stability analysis, we describe the icosahedron by means of the coordinates $\boldsymbol{x}=\left(x_{1}, \ldots, x_{12}\right)$ of its vertices and introduce the Lagrangian

$$
\mathcal{L}=\mathcal{E}-\lambda \mathcal{V}
$$

wherein

$$
\mathcal{V}=\sum_{(k, \ell, m) \in \mathscr{F}}\left|\boldsymbol{x}_{\boldsymbol{k}} \cdot\left(\boldsymbol{x}_{\ell} \times \boldsymbol{x}_{\boldsymbol{m}}\right)\right|
$$

is the volume of the icosahedron, and $\lambda$ is the Lagrange multiplier imposing volume conservation. Let $\boldsymbol{x}^{*}$ denote the coordinates of the platonic icosahedron; imposing $\nabla \mathcal{L}\left(x^{*}, \lambda^{*}\right)=\mathbf{0}$ yields the corresponding value $\lambda^{*}$ of the Lagrange multiplier. The Hessian for this stability problem is $\mathrm{H}=\mathrm{P} \cdot(\nabla \nabla \mathcal{L}) \cdot \mathrm{P}$, where the matrix $\mathrm{P}=\mathrm{I}-\boldsymbol{v} \boldsymbol{v}$ describes the projection onto the kernel of $\boldsymbol{v}=\nabla \mathcal{V}\left(x^{*}, \lambda^{*}\right)$ [34,35]. We note in passing that, since the mobility matrix is invertible and therefore an isomorphism, it does not affect the stability analysis.

\section{Rotator-phase mechanism}

For each face $(k, \ell, m) \in \mathscr{F}$, we define

$$
n_{k \ell m}= \pm\left(x_{k} \times x_{\ell}+x_{\ell} \times x_{m}+x_{m} \times x_{k}\right)
$$

to be its normal, with the sign selecting the outward normal. We note that $\boldsymbol{n}_{\boldsymbol{k} \ell \boldsymbol{m}}$ is not normalized to have unit length. Equation (3) thus becomes

$$
\begin{aligned}
\mathcal{E}_{1}= & \sum_{(k, \ell, m) \in \mathscr{F}} \frac{1}{2}\left\|\boldsymbol{n}_{\boldsymbol{k} \ell \boldsymbol{m}}\right\| \\
& -\alpha \sum_{\substack{(k, \ell, m) \in \mathscr{F} \\
(k, \ell) \in \mathscr{F} \\
m \neq n}}\left\|\boldsymbol{x}_{\boldsymbol{k}}-\boldsymbol{x}_{\ell}\right\| F\left(\delta_{k \ell m n}\right),
\end{aligned}
$$

wherein $\delta_{k \ell m n}$ is defined by

$$
\cos \delta_{k \ell m n}=\frac{\boldsymbol{n}_{k \ell m} \cdot \boldsymbol{n}_{k \ell n}}{\left\|\boldsymbol{n}_{k \ell m}\right\|\left\|\boldsymbol{n}_{k \ell n}\right\|} .
$$

On substituting Eq. (19) into Eq. (16) and differentiating, we obtain an expression for the Hessian $\mathrm{H}_{1}^{*}$ evaluated at the fixed point corresponding to the platonic icosahedron; this expression takes the form

$$
\mathrm{H}_{1}^{*}=\mathrm{S}^{*}-\alpha\left[F\left(\delta^{*}\right) \mathrm{A}^{*}+F^{\prime}\left(\delta^{*}\right) \mathrm{B}^{*}+F^{\prime \prime}\left(\delta^{*}\right) \mathrm{C}^{*}\right],
$$

where the matrices $A^{*}, B^{*}, C^{*}, S^{*}$ are purely geometric, and where $\delta^{*} \approx 138.2^{\circ}$ is the dihedral angle of the platonic icosahedron. We set $F\left(\delta^{*}\right)=1$ without loss of generality; in particular, and as noted earlier, only the local behavior of $F$ near $\delta=\delta^{*}$ thus enters the linear stability analysis (Fig. 5). We sketch the calculations leading to expressions for these matrices in Appendix C.

Evaluating these expressions numerically [36] using MATLAB (The MathWorks, Inc.), we find in particular that $\mathrm{S}^{*}$ is positive semidefinite, so the platonic icosahedron is stable if $\alpha=0$, as expected. The matrices $\mathrm{A}^{*}, \mathrm{~B}^{*}$ are indefinite, but $\mathrm{C}^{*}$ is positive semidefinite. It follows that, for $\alpha>0$, a regular icosahedron is unstable provided that $F^{\prime \prime}\left(\delta^{*}\right)$ is large enough. More generally, the stability boundary for $\alpha>0$ can be computed numerically by a bisection search; results are shown in Fig. 7. Kinks in the curves defining the stability boundary indicate different eigenvalues crossing zero at the stability boundary. We conclude that a regular icosahedron is unstable to small perturbations for appropriate choices of the microscopic law $F$. For a fixed choice of microscopic law, only droplets that are small enough (i.e., have large enough values of $\alpha$ ) deform; larger droplets are stable (Fig. 7). The rotator-phase mechanism can thus explain the deformations away from the initial platonic icosahedron.

The eigenmodes of a hexagonal polygonal droplet, revealed by a similar, yet rather more straightforward linear stability analysis [15], explain the alternative outcomes of quadrilaterals and triangles at later stages of the droplet evolution [15], but the present, more intricate analysis is required to explain how the droplets can flatten and thus reach the hexagonal state in the first place.

The above analysis can be extended to any platonic solid. In the particular case of a regular octahedron, the geometry of the eigenmodes is much simpler, and we therefore discuss these eigenmodes briefly in Appendix D. 


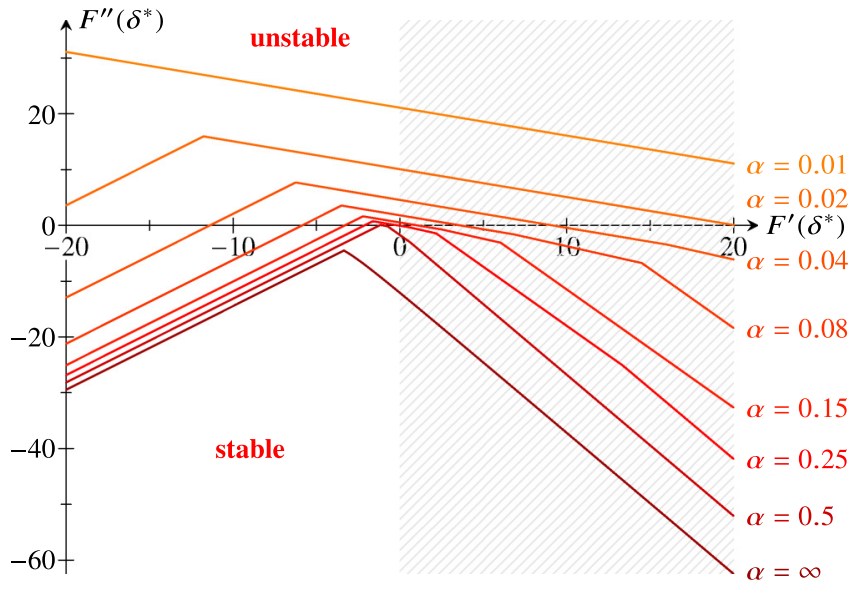

FIG. 7. Stability of the platonic icosahedron for the rotator-phase mechanism. The stability boundary is shown in $\left(F^{\prime}\left(\delta^{*}\right), F^{\prime \prime}\left(\delta^{*}\right)\right)$ space for different values of $\alpha>0$. Only the region with $F^{\prime}\left(\delta^{*}\right)<0$ is expected to be physically relevant.

\section{Elastic buckling mechanism}

For the elastic buckling mechanism (with no buoyancy term), the Hessian $\mathrm{H}_{2}^{*}$ at the platonic icosahedron is obtained by a similar calculation, starting from Eq. (7). We find

$$
\mathrm{H}_{2}^{*}=\mathrm{S}^{*}+\beta \mathrm{D}^{*}
$$

where the matrices $D^{*}, S^{*}$ are purely geometric. Upon numerical evaluation [36], both are found to be positive semidefinite, and hence $\mathrm{H}_{2}^{*}$ is positive semidefinite, too, for any $\beta \geqslant 0$, since the sum of positive semidefinite matrices is positive semidefinite. Accordingly, the regular icosahedron is stable under the elastic buckling mechanism in the absence of buoyancy. The regular icosahedron is not a stationary point of $\mathcal{E}_{2}^{\prime}$, and so will deform if buoyancy is included.

The key conclusion from the stability analysis is therefore that the elastic buckling mechanism can explain the deformations of the initial icosahedron if and only if buoyancy effects become important, i.e., if and only if surface tension drops to ultralow values during cooling. By contrast, the rotatorphase mechanism does not require such a reduction in surface tension to explain these deformations.

\section{B. Droplet flattening}

The linear stability analysis has revealed necessary conditions for the initial regular icosahedron to deform under either mechanism. Larger deformations, and, in particular, flattening, of the polyhedral droplets must however be studied numerically.

The experimental data suggest that the icosahedron flattens symmetrically (Fig. 2), and hence that the four parallel equilateral triangles that define a platonic icosahedron (Fig. 8) remain equilateral during the flattening, although their relative positions with respect to the flattening axis changes. (It is only after the icosahedron has flattened that this symmetry is broken as the polygonal droplets deform. In other words, the component of the initial perturbations of the icosahedron corresponding to these asymmetric deformations is so small that it remains small during the entire flattening process.)

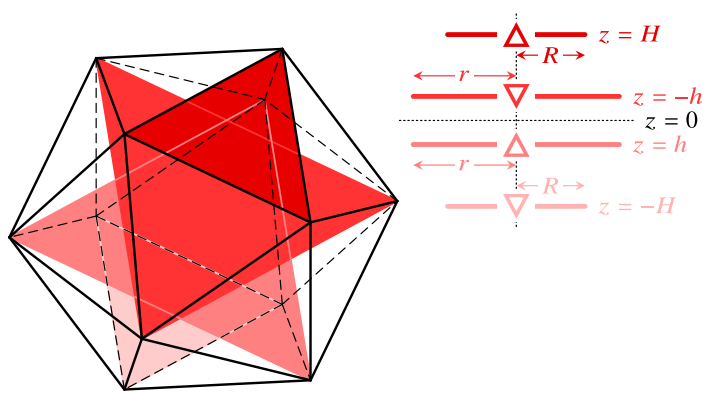

FIG. 8. Simplified model of a symmetric icosahedron. Four parallel equilateral triangles that are symmetric with respect to the midplane of the polyhedron define a symmetric icosahedron in terms of their circumradii $r, R$ and vertical positions $\pm h, \pm H$. The inset shows definitions of variables $r, R, h, H$, and orientations of the four equilateral triangles.

This suggests using a simplified representation of a symmetric icosahedron, defined by four parallel equilateral triangles (Fig. 8), for the numerical calculations. Such an icosahedron is defined in terms of four variables (Fig. 8, inset); one of these can be eliminated using the volume conservation constraint (Appendix E).

We solve Eq. (14) governing the deformations of the icosahedron numerically using the stiff solver ode15s of MATLAB (The MathWorks, Inc.).

\section{Rotator-phase mechanism}

We begin by considering the rotator-phase mechanism and the limit $\alpha=\infty$ where the tendency to form rotator phase swamps the stabilizing effect of surface tension. Our first observation is that a (slightly perturbed platonic) symmetric icosahedron may indeed flatten into an octahedron [Fig. 9(a)] under the rotator-phase mechanism: As the icosahedron flattens, the top and bottom equilateral triangles expand faster than the middle ones, leading to six pairs of vertices merging to yield a (nonplatonic) octahedron, in qualitative agreement with the shape evolution seen in experiments. This evolution depends on the choice of the microscopic law $F(\delta)$ and the initial perturbation. As far as the choice of $F(\delta)$ is concerned, flattening occurs, for example, for $F(\delta) \propto \pi^{3}-\delta^{3}$ [Fig. 9(a)], but does not occur for the simplest (linear) law in agreement with conditions (5), $F(\delta) \propto \pi-\delta$ [Fig. 9(b)]. Flattening is not observed either if the dependence on the dihedral angle is not included. We have checked that the behavior in Fig. 9(a) is representative of the behavior observed for sufficiently concave choices of the microscopic law (Fig. 5). In this way, the analysis constrains the global functional form of $F(\delta)$ beyond conditions (5). As far as the initial perturbations are concerned, flattening similarly occurs for some, but not all, perturbations of the regular icosahedron [Figs. 9(a) and 9(c)].

To explore the latter effect and the role of surface tension, we consider relative perturbations, of fixed magnitude $\varepsilon$, of the parameters defining the symmetric icosahedron (Fig. 8, inset). Taking the initial perturbations $\delta r, \delta R, \delta H$ of the three parameters $r, R, H$ as the basis for these perturbations without loss of generality, we map the shape outcomes for different initial perturbations onto the surface of a sphere [Fig. 9(d)]. 
(a)

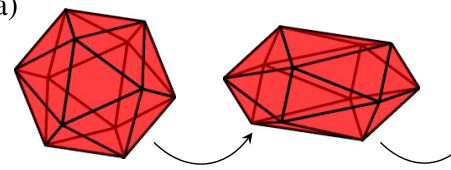

(b)

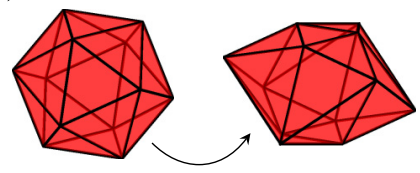

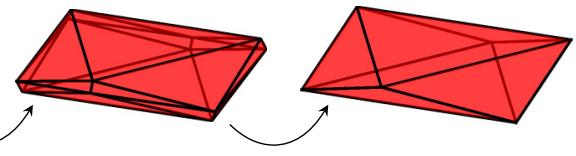

(c)

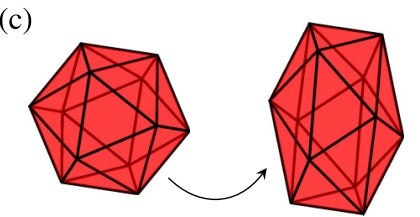

(d)

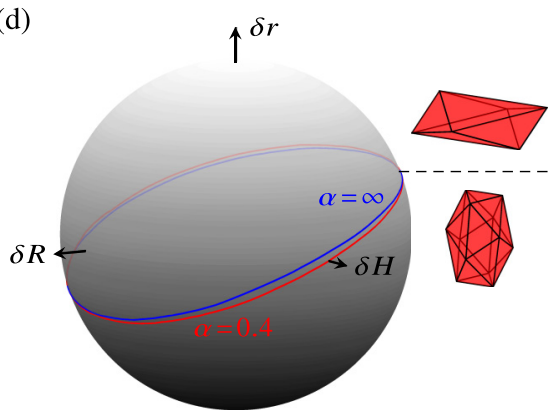

FIG. 9. Flattening of a symmetric icosahedron under the rotator-phase mechanism. (a) Snapshots of a slightly perturbed platonic icosahedron flattening into a (nonplatonic) octahedron, for $\alpha=\infty$, and for the microscopic law $F(\delta) \propto \pi^{3}-\delta^{3}$. (b) Role of the microscopic law: For a different microscopic law, here $F(\delta) \propto \pi-\delta$, but the same initial perturbation, the icosahedron does not flatten into an octahedron. (c) Role of the initial perturbation: For the same microscopic law, but a different initial perturbation, the icosahedron does not flatten either. (d) Three of the four parameters defining the symmetric icosahedron (Fig. 8, inset) can be chosen as a basis for perturbations away from the regular icosahedron. Shape outcomes for such perturbations of fixed magnitude $\varepsilon=0.02$ are then mapped onto the surface of a sphere. The boundary between those initial relative perturbations leading to flattening into an octahedron and those that do not lead to flattening are shown for $F(\delta) \propto \pi^{3}-\delta^{3}$ and different values of $\alpha$.

A boundary divides those initial perturbations that lead to flattening to those that do not, and we observe that this boundary does not strongly depend on $\alpha$ [Fig. 9(d)]. Physically, the initial perturbation is set by the buoyancy of the droplets, and we therefore expect the physically relevant initial perturbations of the droplets to be those with $\delta H<0$ and $\delta r, \delta R>0$, for which the droplet indeed flattens into an octahedron [Fig. 9(d)].

We conclude that, for an appropriate choice of microscopic law, our model of the rotator-phase mechanism predicts flattening in qualitative agreement with the experimental observations for physical initial perturbations of the icosahedron.

\section{Elastic buckling mechanism}

The stability analysis in the previous section has revealed that the regular icosahedron is a stable fixed point if buoyancy does not play a role, Bo $=0$. We therefore consider the case $\mathrm{Bo}>0$, in which the regular icosahedron is, as noted previously, no longer a fixed point of the energy. We begin by analyzing the limit of low surface tension, in which $\beta$, Bo $\gg 1$ and the dynamics depend on the single parameter $B=\mathrm{Bo} / \beta$. Numerically, we find that there is an intermediate range, $B_{-}<B<B_{+}$, in which the platonic icosahedron flattens into an octahedron [Fig. 10(a)]. We estimate $B_{-} \approx 7$ and $B_{+} \approx 140$. If $B<B_{-}$, the droplet settles into a steady state before reaching an octahedral shape [Fig. 10(b)]. If $B>B_{+}$, the three faces adjacent to the top face flatten into its plane [Fig. 10(c)]. Thus, the droplet evolves into a hexagonal prism as the top triangle continues to expand. This is inconsistent with the experimental observation that the top plane remains triangular [Fig. 2(c)], and does not become hexagonal until the end of the flattening.

Qualitatively similar results are obtained at nonzero surface tension; because of its stabilizing effect, $B_{-}$increases with increasing surface tension, i.e., $B_{-} \gtrsim 7$. We conclude that the elastic buckling mechanism predicts flattening in qualitative agreement with the experimental observations if buoyancy effects are neither too strong nor too weak.
To obtain more quantitative estimates, we notice the scaling $B \sim \Delta \rho R^{11 / 3} / E h^{8 / 3}$, wherein, as before, $E$ is the elastic modulus of the frozen surfactant monolayer and $h$ is its thickness, $g \approx 9.81 \mathrm{~m} / \mathrm{s}^{2}$ is the acceleration due to gravity, $R$ is the droplet radius, and $\Delta \rho \approx 250 \mathrm{~kg} / \mathrm{m}^{3}$ is the density difference between the water and oil phases. Hence the bending modulus of the frozen surfactant layer is [27]

$$
K=E h^{3} \sim \frac{\Delta \rho g R^{11 / 3} h^{1 / 3}}{B} .
$$

Usefully, this allows us to obtain an upper bound on $K$ without having to estimate the surface tension, which does not appear in this expression: Denote by $R_{-}$the radius, corresponding to $B_{-}$, of the smallest droplet that can flatten; previous work [5] has shown $R_{-}<2 \mu \mathrm{m}$. Taking $h \approx 2 \mathrm{~nm}$ [28] and using $B_{-}>7$, we obtain that $K<6 \times 10^{-22} \mathrm{~J}$ is required for flattening into an octahedron. By contrast, direct measurements of the bending moduli of shape-shifting droplets in Ref. [16] led to the lower bound $K>10^{3} \mathrm{k}_{\mathrm{B}} \mathrm{T} \gtrsim 3 \times 10^{-18} \mathrm{~J}$, more than three orders of magnitude above the present upper bound.
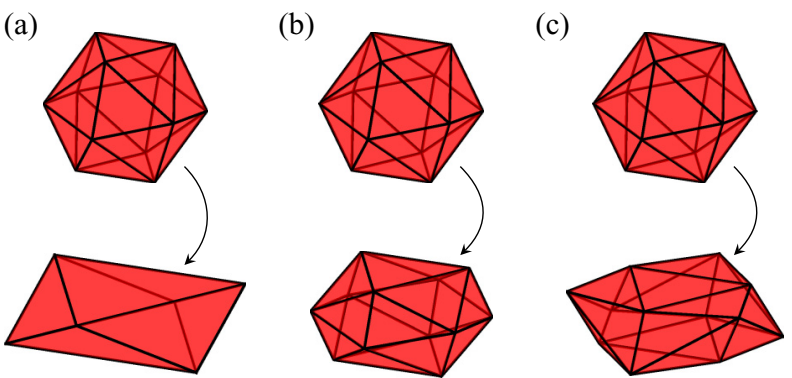

FIG. 10. Flattening of a symmetric icosahedron under the elastic buckling mechanism for different values of $B=\mathrm{Bo} / \beta$. (a) Flattening of a platonic icosahedron into a (nonplatonic) octahedron. (b) If $B$ is too small, the icosahedron settles into an unflattened steady state. (c) If $B$ is too large, the faces connected to the top face flatten into its plane, inconsistent with experimental observations. 


\section{CONCLUSION}

In this paper, we have analyzed the flattening of shapeshifting droplets experimentally and theoretically. Models of the two candidate mechanisms have reproduced the evolution of an icosahedral droplet into a flattened octahedral shape in qualitative agreement with the experimental observations. The elastic buckling mechanism, however, can only reproduce the experimental observations if surface tension decreases by at least four orders of magnitude during the cooling, so that the flattening is driven by a competition between buoyancy and elasticity. Moreover, the resulting estimate of the bending modulus of the surfactant adsorption layer is three orders of magnitude below the values measured in experiments. All of this strongly indicates that the "shape-shifting" droplet phenomena are driven by formation of a rotator phase rather than elastic buckling of the frozen surfactant adsorption layer at ultralow surface tension.

Such ultralow values of surface tension are not, however, thermodynamically forbidden (although systems with negative surface tensions should be catastrophically unstable). While this analysis shows that the shape-shifting deformations observed thus far $[5,8,9,11,18,19]$ are not consistent with the elastic buckling mechanism, there may therefore be other systems to be discovered in which the shape shifting is indeed driven by the interplay of buoyancy and elasticity predicted by this mechanism.

While the simple models used in this paper to represent the droplets as true polyhedra could thus reproduce the experimental flattening dynamics qualitatively, it is important to recognize that the faces of the actual droplets do not remain flat, but deform due to elasticity and surface tension. These simple models do not take into account either the dynamics of elastic defects in the surface and interactions between defects. Defect-defect repulsion has been studied by others, for example in Refs. [4,37-39]. Nevertheless, repeating the computations in this paper for deformable faceted elastic surfaces with defects presents a formidable numerical challenge. Fully resolving the defect energetics would also make possible a more detailed analysis of the very latest stages of the flattening, when the defects become close to each other before possibly merging, and, as discussed earlier, the models derived here break down for defect-defect interactions can therefore no longer be ignored. Experimentally, this breakdown of the model is heralded by the observation (Appendix A) that the latest stages of the flattening depend on the details of the system.

Some questions more specific to the rotator-phase mechanism also remain open: Our analysis has revealed the importance of the dependence of the phase transition on the dihedral angle, expressed by the microscopic law $F$, but how does this dependence on the dihedral angle relate to the fundamental properties of the rotator phase and the phase transition? Indeed, our present approach of choosing a functional form ad hoc for lack of knowledge about the detailed physics involved leaves a certain amount of arbitrariness in the analysis, even though, as noted previously, this is mitigated by the fact that the qualitative behavior of the model does not depend strongly on these details. While deriving this microscopic law from first principles would remove this arbitrariness, this

problem of understanding these microscopic principles is, as pointed out earlier, wholly separate from the question of possible behaviors of the model that we have answered in this paper. We close by noting that the impact of the kinetics of phase change on the droplet evolution also remains unclear.

\section{ACKNOWLEDGMENTS}

We thank Slavka Tcholakova for numerous fruitful discussions and for helping with the analysis of the microscopy data. We also thank Ireth García-Aguilar for a discussion of her unpublished work [39] on shape-shifting droplets, and are grateful for support from the European Research Council (Grant EMATTER 280078 to S.K.S.), the Engineering and Physical Sciences Research Council (Established Career Fellowship No. EP/M017982/1 to R.E.G.; Fellowship No. EP/R028915/1 to S.K.S.; Doctoral Prize Fellowship to P.A.H.), the Schlumberger Chair Fund, and Magdalene College, Cambridge (Nevile Research Fellowship to P.A.H.).

\section{APPENDIX A: EXPERIMENTAL METHODS AND DETAILS}

In this Appendix, we provide details on the experimental methods (Fig. 11) and on the system dependence of the final step of the droplet flattening.

\section{Description of the experimental setup}

Oil emulsion droplets were observed in glass capillaries placed inside a thermostatic chamber [Fig. 11(a)] below the objective of the microscope [Fig. 11(b)], as described previously $[5,9]$. Observations were performed with an upright optical microscope in reflected white light. Due to the buoyancy force, oil droplets float just below the upper wall of the glass capillary [Fig. 11(b)]. The aqueous film formed between this wall and the upper surface (closest to the capillary) of the droplet was observed in reflected light.

\section{System dependence of droplet flattening}

As mentioned in the main text, the details of the final step of the droplet flattening depend on the emulsion system: For those systems in which no asperities (long cylindrical protrusions from the platelet corners) are formed, i.e., in group B systems according to the classification of Ref. [9], pairs

(a)

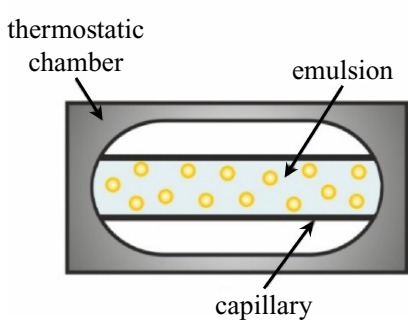

(b)

FIG. 11. Experimental setup for reflected-light microscopy of droplet deformations. (a) Emulsions are examined in a glass capillary placed inside a thermostatic chamber for microscopic observations during cooling. (b) Side view: Due to buoyancy, oil droplets float just below the top surface of the capillary, under the objective of the microscope. 

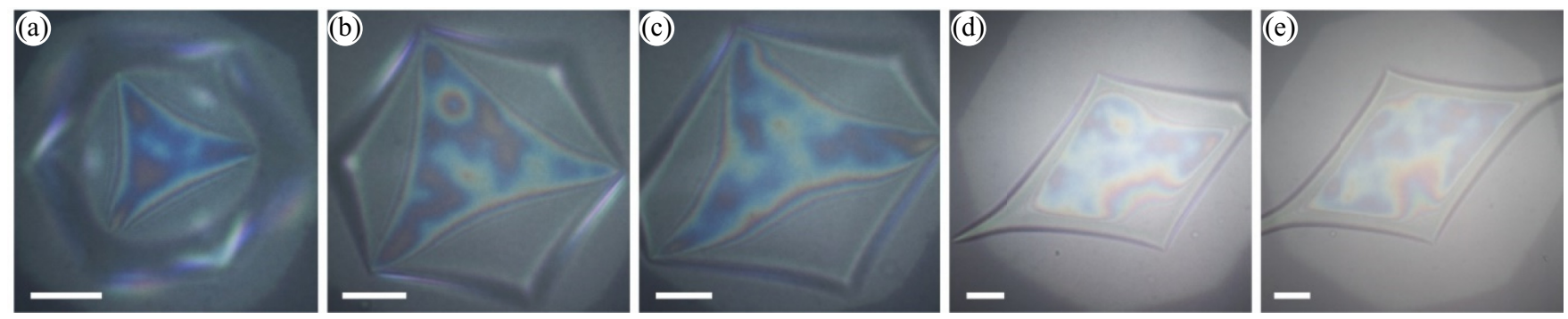

FIG. 12. Merging of the vertices of a polyhedral drop with formation of asperities. This experiment was performed with a hexadecane emulsion droplet, immersed in $1.5 \mathrm{wt}$ \% Brij 58 surfactant solution. Merging of vertices causes the octahedral stage (b) to disproportionate (c), leading to the formation of a tetragonal platelet with asperities (e). Scale bars: $10 \mu \mathrm{m}$.

of vertices eventually merge to form true hexagonal platelets [Fig. 2(e)]. By contrast, in those systems in which asperities do form, i.e., in groups $\mathrm{A}$ and $\mathrm{C}$ of Ref. [9], the merging of the vertices also drives the transition from an octahedral droplet into tetragonal or triangular plates (Fig. 12).

\section{APPENDIX B: CLASSIFICATION OF A FAMILY OF POLYHEDRA}

In this Appendix, we classify polyhedra that can be obtained by edge contraction from an icosahedron. Starting from an icosahedron, we thus contract edges to reduce the number of vertices of the polyhedron. Representing each polyhedron by its undirected edge graph $\mathscr{G}=(\mathscr{V}, \mathscr{E})$ of vertices $\mathscr{V}$ and edges $\mathscr{E}$, we classify, numerically and up to isomorphism, the 105 graphs of at least six vertices obtained by edge contraction in this way. (Such a classification is of course a hard problem in general, but the graphs are small enough for classification by brute force to be straightforward.) Requiring these graphs to correspond to true polyhedra, we require that any edge be adjacent to exactly two faces, and that, for any $v \in \mathscr{V}, \mathscr{G} \backslash v$ be connected. Geometrically, these conditions ensure that there are no loose planes or segments, and that the polyhedron is not the union of two smaller polyhedra glued together at a vertex or along an edge. Physically, such deformations would strongly cinch in the droplet surface, and

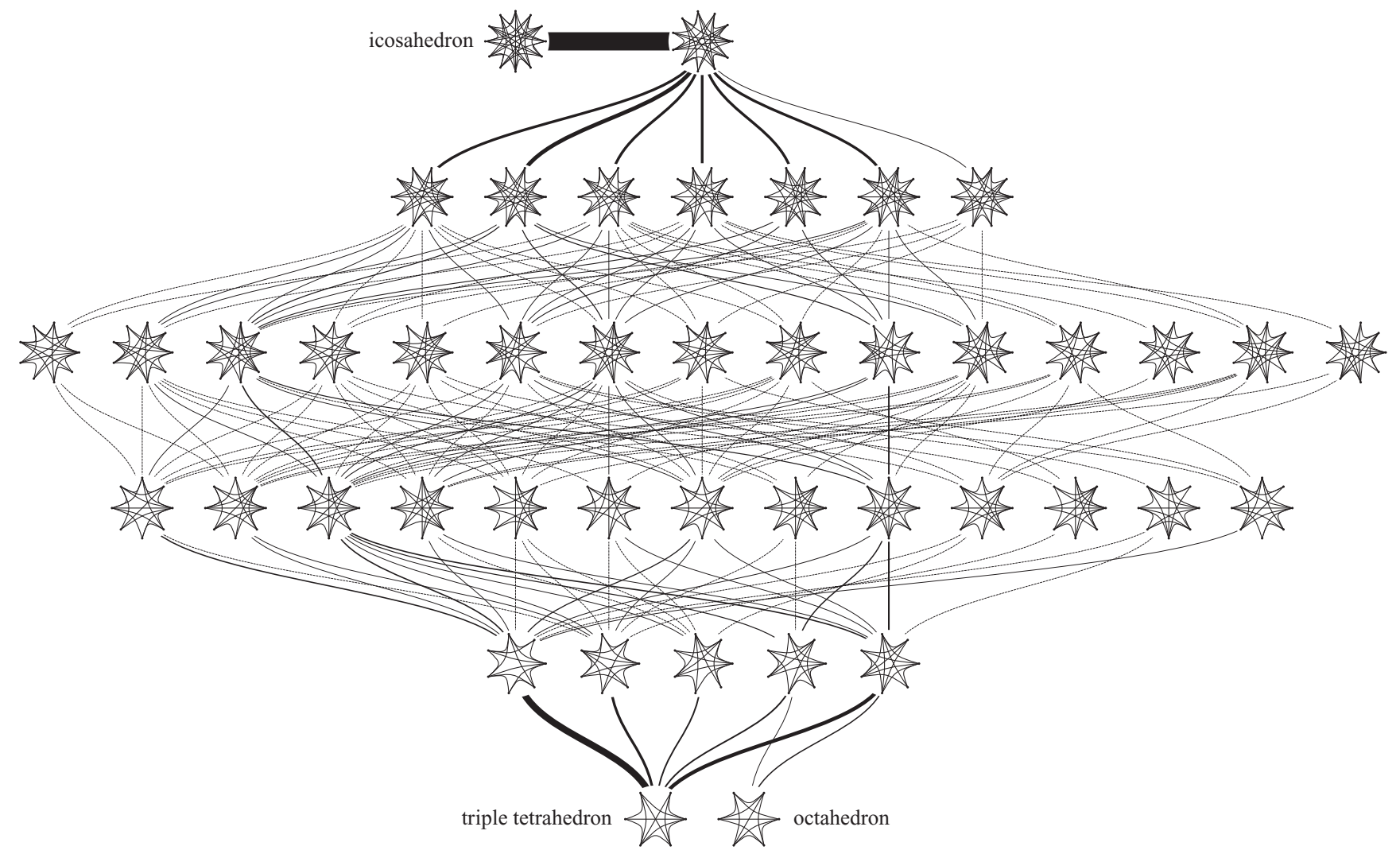

FIG. 13. Classification of polyhedra of a least six vertices obtained by edge contraction from an icosahedron. Edge graphs of the 44 polyhedra obtained are shown, with lines between polyhedra indicating possible edge contraction paths. Linewidths are proportional to the probability that a random walk starting from the icosahedron passes through a particular transition. Small probabilities are represented by dotted lines. The icosahedron, octahedron, and triple tetrahedron are labeled. 
are thus energetically unfavourable. Upon discarding graphs not satisfying this conditions, we are left with 44 polyhedra.

The edge graphs of these 44 polyhedra are shown in Fig. 13. In particular, we find that there are only two possible polyhedra on six vertices: an octahedron and a triple tetrahedron (that is, three tetrahedra glued together along their faces). Interestingly, random edge contractions from the icosahedron result in an octahedron with approximate probability 0.11 only, compared to 0.89 for the triple tetrahedron. Formation of a triple tetrahedron requires additional symmetry breaking, though. For this reason, and from this purely combinatorial analysis, the octahedron is to be expected as an intermediate step in the evolution of the icosahedron.

\section{APPENDIX C: DETAILS OF THE LINEAR STABILITY CALCULATION}

In this Appendix, we sketch the derivation of expressions for the different terms that appear in Eq. (21). Substituting Eq. (19) into expression (16) for the Lagrangian, and differentiating,

$$
\begin{aligned}
\frac{\partial \mathcal{L}_{1}}{\partial \boldsymbol{x}_{\boldsymbol{i}}} & =\boldsymbol{s}_{\boldsymbol{i}}-\alpha\left(f_{*} \boldsymbol{a}_{\boldsymbol{i}}+f_{*}^{\prime} \boldsymbol{b}_{\boldsymbol{i}}\right)-\lambda^{*} \boldsymbol{v}_{\boldsymbol{i}}, \\
\frac{\partial^{2} \mathcal{L}_{1}}{\partial \boldsymbol{x}_{\boldsymbol{i}} \partial \boldsymbol{x}_{\boldsymbol{j}}} & =\mathrm{S}_{i j}-\alpha\left(f_{*} \mathrm{~A}_{i j}+f_{*}^{\prime} \mathrm{B}_{i j}+f_{*}^{\prime \prime} \mathrm{C}_{i j}\right)-\lambda^{*} \mathrm{~V}_{i j},
\end{aligned}
$$

where $f_{*}=F\left(\delta^{*}\right), f_{*}^{\prime}=F^{\prime}\left(\delta^{*}\right), f_{*}^{\prime \prime}=F^{\prime \prime}\left(\delta^{*}\right)$, and where the vectors $\boldsymbol{s}_{\boldsymbol{i}}, \boldsymbol{a}_{\boldsymbol{i}}, \boldsymbol{b}_{\boldsymbol{i}}, \boldsymbol{v}_{\boldsymbol{i}}$ and matrices $\mathrm{S}_{i j}, \mathrm{~A}_{i j}, \mathrm{~B}_{i j}, \mathrm{C}_{i j}, \mathrm{~V}_{i j}$ can be expressed as sums of simpler expressions, obtaining which is a mere lengthy and unpleasant exercise in differentiating vectors and their products. Solving for $\lambda^{*}$ using Eq. (C1) and one component of $\partial \mathcal{L}_{1} / \partial \boldsymbol{x}=\mathbf{0}$ yields

$$
\lambda^{*}=s^{*}-\alpha\left(a^{*} f_{*}+b^{*} f_{*}^{\prime}\right),
$$

where $s^{*}, a^{*}, b^{*}$ are scalars. We check post facto that this choice of $\lambda^{*}$ indeed leads to all 36 components of $\partial \mathcal{L}_{1} / \partial \boldsymbol{x}$ vanishing. Hence Eq. (C2) becomes

$$
\frac{\partial^{2} \mathcal{L}_{1}}{\partial \boldsymbol{x}_{\boldsymbol{i}} \partial \boldsymbol{x}_{\boldsymbol{j}}}=\mathrm{S}_{i j}^{*}-\alpha\left(f_{*} \mathrm{~A}_{i j}^{*}+f_{*}^{\prime} \mathrm{B}_{i j}^{*}+f_{*}^{\prime \prime} \mathrm{C}_{i j}^{*}\right),
$$

wherein

$$
\begin{array}{ll}
\mathrm{S}_{i j}^{*}=\mathrm{S}_{i j}-s^{*} \mathrm{~V}_{i j}, & \mathrm{~A}_{i j}^{*}=\mathrm{A}_{i j}-a^{*} \mathrm{~V}_{i j}, \\
\mathrm{~B}_{i j}^{*}=\mathrm{B}_{i j}-b^{*} \mathrm{~V}_{i j}, & \mathrm{C}_{i j}^{*}=\mathrm{C}_{i j} .
\end{array}
$$

On assembling these matrices into four $36 \times 36$ matrices made of these $3 \times 3$ blocks and projecting these onto the kernel of $\boldsymbol{v}=\boldsymbol{\nabla} \mathcal{V}\left(\boldsymbol{x}^{*}, \lambda^{*}\right)$ using $\mathrm{P}=\mathrm{I}-\boldsymbol{v} \boldsymbol{v}$, we obtain Eq. (21).

\section{APPENDIX D: EIGENMODES OF A REGULAR OCTAHEDRON}

In this Appendix, we discuss the linear stability analysis of a regular octahedron. Expanding about the platonic octahedron, $\mathrm{H}_{1}^{*}=\mathrm{S}^{*}-\alpha\left[F\left(\delta^{*}\right) \mathrm{A}^{*}+F^{\prime}\left(\delta^{*}\right) \mathrm{B}^{*}+F^{\prime \prime}\left(\delta^{*}\right) \mathrm{C}^{*}\right]$, as in Eq. (21), where $\delta^{*} \approx 109.5^{\circ}$ is now the dihedral angle of a platonic octahedron, and where the purely geometric matrices $A^{*}, B^{*}, C^{*}, S^{*}$ are found numerically. What makes the case of the regular octahedron simpler is the fact that $A^{*}, B^{*}, C^{*}, S^{*}$ commute pairwise, and hence can be diagonalized simultaneously [40]. (a)

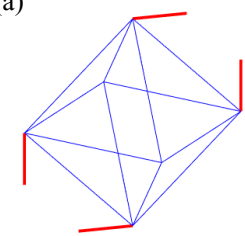

(c)

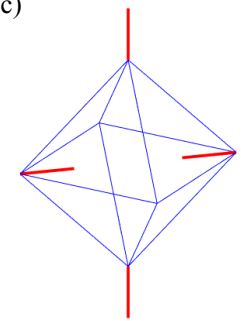

(b)

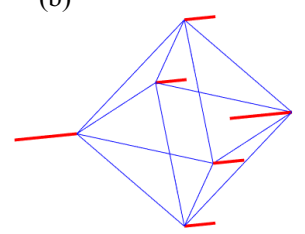

(d)

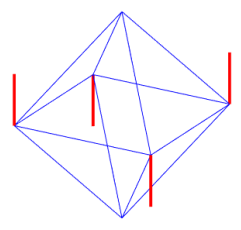

FIG. 14. Nontrivial eigenmodes of a regular octahedron. The dimensions of the four eigenspaces are: (a) $\operatorname{dim}=3$, (b) $\operatorname{dim}=3$, (c) $\operatorname{dim}=2$, (d) $\operatorname{dim}=3$.

Simultaneous diagonalizability means that stability boundaries can be computed analytically as the intersection of planes, but here we shall merely point out that the simultaneous eigenmodes are geometrically "nice." Indeed, of the $6 \times 3=18$ simultaneous eigenmodes [41], 7 are neutral modes, corresponding to three rotations, three translations, and a scaling mode (the latter is neutral since it is not volumepreserving). The remaining 11 eigenmodes divide into four eigenspaces for which bases aligned with the symmetry axes and planes of the octahedron can be picked, as shown in Fig. 14.

It is natural to wonder whether there is a deeper reason for this simplification in the case of the octahedron. We do not have an answer to this question, but note that eigenmodes must respect the symmetries of the polyhedron. It is therefore tempting to speculate that, in the case of the octahedron, the existence of a common eigenbasis is caused by the fact that there are simply not enough eigenmodes that are available (i.e., allowed by the symmetries of the octahedron).

\section{APPENDIX E: SYMMETRIC ICOSAHEDRON MODEL}

In this Appendix, we derive the volume conservation constraint for the symmetric icosahedron model. Up to scaling, we may take $r^{*}=1$ for the regular icosahedron. Using an explicit coordinate representation of the icosahedron, we then obtain

$$
R^{*}=\frac{\sqrt{5}-1}{2}, \quad H^{*}=\frac{\sqrt{5}+1}{4}, \quad h^{*}=-\frac{3-\sqrt{5}}{4},
$$

with coordinates $r, R, H, h$ defined as in the inset of Fig. 8 . The volume conservation constraint thus takes the form

$$
\left[(r+R)^{2}+r^{2}\right] H-R(2 r-R) h=\frac{5}{2}(\sqrt{5}-1) .
$$

We use this relation to eliminate $h$. Next, using MATHEMATICA (Wolfram, Inc.), we derive expressions for the coefficients of the mobility matrix, from Eq. (15), and for the energy gradient. These expressions, albeit too large to reproduce here, are easily evaluated numerically and given in the Supplemental Material [36]. 
[1] Euclid, $\Sigma \tau \circ \iota \chi \varepsilon \tau \tilde{\alpha} \alpha$ (Ancient Greece), Book XIII; for a modern translation, see, e.g., T. L. Heath, Euclid's Elements, edited by D. Densmore (Green Lion Press, Santa Fe, NM, 2002).

[2] M. J. Bowick and R. Sknepnek, Pathways to faceting of vesicles, Soft Matter 9, 8088 (2013).

[3] H. S. Seung and D. R. Nelson, Defects in flexible membranes with crystalline order, Phys. Rev. A 38, 1005 (1988).

[4] M. J. Bowick, D. R. Nelson, and A. Travesset, Interacting topological defects on frozen topographies, Phys. Rev. B 62, 8738 (2000).

[5] N. Denkov, S. Tcholakova, I. Lesov, D. Cholakova, and S. K. Smoukov, Self-shaping of oil droplets via the formation of intermediate rotator phases upon cooling, Nature (London) $\mathbf{5 2 8}$, 392 (2015).

[6] E. Sloutskin, C. D. Bain, B. M. Ocko, and M. Deutsch, Surface freezing of chain molecules at the liquid-liquid and liquid-air interfaces, Faraday Discuss. 129, 339 (2005), and in particular p. 363 of the corresponding general discussion, ibid. 129, 353 (2005)

[7] K. Golemanov, S. Tcholakova, N. D. Denkov, and T. Gurkov, Selection of surfactants for stable paraffin-in-water dispersions, undergoing solid-liquid transition of the dispersed particles, Langmuir 22, 3560 (2006).

[8] N. Denkov, D. Cholakova, S. Tcholakova, and S. K. Smoukov, On the mechanism of drop self-shaping in cooled emulsions, Langmuir 32, 7985 (2016).

[9] D. Cholakova, N. Denkov, S. Tcholakova, I. Lesov, and S. K. Smoukov, Control of drop shape transformations in cooled emulsions, Adv. Colloid Interface Sci. 235, 90 (2016).

[10] S. Tcholakova, Z. Valkova, D. Cholakova, Z. Vinarov, I. Lesov, N. Denkov, and S. K. Smoukov, Efficient self-emulfication via cooling-heating cycles, Nat. Commun. 8, 15012 (2017).

[11] D. Cholakova, Z. Valkova, S. Tcholakova, N. Denkov, and S. K. Smoukov, "Self-shaping" of multicomponent drops, Langmuir 33, 5696 (2017).

[12] Z. Valkova, D. Cholakova, S. Tcholakova, N. Denkov, and S. K. Smoukov, Mechanisms and control of self-emulsification upon freezing and melting of dispersed alkane drops, Langmuir 33, 12155 (2017).

[13] I. Lesov, Z. Valkova, E. Vassileva, G. S. Georgiev, K. Ruseva, M. Simeonov, S. Tcholakova, N. D. Denkov, and S. K. Smoukov, Bottom-up synthesis of polymeric micro- and nanoparticles with regular anisotropic shapes, Macromolecules 51, 7456 (2018).

[14] D. Cholakova, N. Denkov, S. Tcholakova, Z. Valkova, and S. K. Smoukov, Multilayer formation in self-shaping emulsion droplets, Langmuir 35, 5484 (2019).

[15] P. A. Haas, R. E. Goldstein, S. K. Smoukov, D. Cholakova, and N. Denkov, Theory of Shape-Shifting Droplets, Phys. Rev. Lett. 118, 088001 (2017).

[16] S. Guttman, Z. Sapir, M. Schultz, A. V. Butenko, B. M. Ocko, M. Deutsch, and E. Sloutskin, How faceted liquid droplets grow tails, Proc. Natl. Acad. Sci. USA 113, 493 (2016).

[17] S. Guttman, B. M. Ocko, M. Deutsch, and E. Sloutskin, From faceted vesicles to liquid icoshedra: Where topology and crystallography meet, Curr. Opin. Colloid Interface Sci. 22, 35 (2016).

[18] S. Guttman, Z. Sapir, B. M. Ocko, M. Deutsch, and E. Sloutskin, Temperature-tuned faceting and shape changes in liquid alkane droplets, Langmuir 33, 1305 (2017).
[19] O. Marin, M. Alesker, S. Guttman, G. Gershinsky, E. Edri, H. Shpaisman, R. E. Guerra, D. Zitoun, M. Deutsch, and E. Sloutskin, Self-faceting of emulsion droplets as a route to solid icosahedra and other polyhedra, J. Colloid Interface Sci. 538, 541 (2019).

[20] S. Guttman, E. Kesselman, A. Jacob, O. Marin, D. Danino, M. Deutsch, and E. Sloutskin, Nanostructures, faceting, and splitting in nanoliter to yoctoliter liquid droplets, Nano Lett. 19, 3161 (2019).

[21] N. Martin, K. P. Sharma, R. L. Harniman, R. M. Richardson, R. J. Hutchings, D. Alibhai, M. Li, and S. Mann, Lightinduced dynamic shaping and self-division of multipodal polyelectrolyte-surfactant microarchitectures via azobenzene photomechanics, Sci. Rep. 7, 41327 (2017).

[22] E. B. Sirota and A. B. Herhold, Transient phase-induced nucleation, Science 283, 529 (1999).

[23] D. Cholakova and N. Denkov, Rotator phases in alkane systems: In bulk, surface layers and micro/nano-confinements, Adv. Colloid Interface Sci. 269, 7 (2019).

[24] This assumes the rotator and liquid phases to have equal densities, but, as already noted in Ref. [15], including density differences would not fundamentally change the analysis.

[25] J. Lidmar, L. Mirny, and D. R. Nelson, Virus shapes and buckling transitions in spherical shells, Phys. Rev. E 68, 051910 (2003).

[26] A. E. Lobkovsky, Boundary layer analysis of the ridge singularity in a thin plate, Phys. Rev. E 53, 3750 (1996).

[27] Reference [16] suggested that continuum elasticity breaks down for the shape-shifting droplets, based on estimates involving the Föppl-von Kármán (FvK) number $\Gamma \sim Y R^{2} / K$, where $Y, K$ are the stretching and bending moduli of the surface of the droplets, and $R$ is, as before, their radius. However, Ref. [16] approximated $\Gamma \approx \Gamma_{1}$, where $\Gamma_{1} \approx 150$ is the critical FvK number for defect buckling [3], while Ref. [25] shows that icosahedral buckling occurs only for $\Gamma>\Gamma_{2} \gg \Gamma_{1}$. Here, we note the scalings $Y \sim E h, K \sim E h^{3}$ of continuum elasticity, wherein $E$ is the elastic modulus. Thus $Y / K \sim h^{2}$ and $\Gamma \sim(R / h)^{2}$. With $h \approx 2 \mathrm{~nm}[28]$ and $10^{4}<\Gamma_{2}<10^{7}$ [25], this implies a minimal radius $R_{2}$ for formation of an icosahedron, $0.2 \mu \mathrm{m}<R_{2}<6.5 \mu \mathrm{m}$, in agreement with experiments and other estimates [5,16], although very recent work has suggested that somewhat smaller droplets with $R \approx 50 \mathrm{~nm}$ can also form icosahedra [20]. We are therefore justified in using the elastic scalings for the energy of an elastic ridge and justified in using $K \sim E h^{3}$ in Eq. (23).

[28] E. Sloutskin, Z. Sapir, C. D. Bain, Q. Lei, K. M. Wilkinson, L. Tamam, M. Deutsch, and B. M. Ocko, Wetting, Mixing, and Phase Transitions in Langmuir-Gibbs Films, Phys. Rev. Lett. 99, 136102 (2007)

[29] W. H. Hager, Wilfrid Noel Bond and the Bond number, J. Hydraul. Res. 50, 3 (2012).

[30] At standard conditions, alkanes with 14-20 carbon atoms have densities of about $750 \mathrm{~kg} / \mathrm{m}^{3}$; see, e.g., Handbook of Chemistry and Physics, 98th ed., edited by J. R. Rumble (CRC Press, Boca Raton, FL, 2017), Chap. 3.

[31] Consider indeed a regular $n$-gon of area $A_{0}$ and perimeter $P_{0}$, and suppose to the contrary that there is a $n$-gon of area $A_{0}$ and perimeter $P_{1}<P_{0}$. Shrinking the regular $n$-gon, there thus exist a regular $n$-gon of area $A_{1}<A_{0}$ and perimeter $P_{1}$, and 
a $n$-gon of area $A_{0}$ and perimeter $P_{1}$. This is a contradiction, for among all $n$-gons of fixed perimeter, the regular $n$-gon has the largest area; see, e.g., V. M. Tikhomirov, in Stories about Maxima and Minima, Mathematical World Vol. 1 (American Mathematical Society, Providence, RI, 1991), Chap. 2, pp. 9-18.

[32] Jensen's inequality [see, e.g., D. Stirzaker, in Elementary Probability, 2nd ed. (Cambridge University Press, Cambridge, UK, 2003), Chap. 4.6, pp. 131-134] implies that the elastic energy of a nonregular polygon is lower than that of a regular polygon of the same perimeter, as the elastic energy is a concave function of edge length. However, this does not quite imply that disproportionation of side lengths is energetically favorable since a nonregular polygon of the same area must have a larger perimeter than the original regular polygon [31]. That disproportionation is indeed favorable can be proved by a linear stability analysis similar to the analysis in Sec. IV A which therefore confirms the qualitative convexity argument.

[33] Z. Suo, Motions of microscopic surfaces in materials, in $\mathrm{Ad}$ vances in Applied Mechanics, edited by J. W. Hutchinson and T. Y. Wu (Elsevier, New York, NY, 1997), Vol. 33, pp. 193-294.

[34] C. D. Meyer, in Matrix Analysis and Applied Linear Algebra (Society for Industrial and Applied Mathematics, Philadelphia, PA, 2000), Chaps. 5.6 and 5.9, pp. 320-340 and 383-393.
[35] R. K. Sundaram, in A First Course in Optimization Theory (Cambridge University Press, Cambridge, UK, 1996), Chap. 5, pp. 112-144.

[36] See Supplemental Material at http://link.aps.org/supplemental/ 10.1103/PhysRevResearch.1.023017 for (i) numerical values of the matrices in the linear stability analysis and (ii) exact expressions of the components of the mobility matrix and energy gradient for the symmetric icosahedron model.

[37] G. M. Grason, Defects in crystalline packings of twisted filament bundles. I. Continuum theory of disclinations, Phys. Rev. E 85, 031603 (2012).

[38] S. Li, P. Roy, A. Travesset, and R. Zandi, Why large icosahedral viruses need scaffolding proteins, Proc. Natl. Acad. Sci. USA 115, 10971 (2018).

[39] I. García-Aguilar, P. Fonda, L. Giomi, and E. Sloutskin, Defect screening in faceted emulsion droplets (unpublished).

[40] R. A. Horn and C. R. Johnson, in Matrix Analysis (Cambridge University Press, Cambridge, UK, 1985), Chap. 1.3, pp. 44-56.

[41] Simultaneous eigenmodes and eigenvalues were computed using the algorithm described in A. Bunse-Gerstner, R. Byers, and V. Mehrmann, Numerical methods for simultaneous diagonalization, SIAM J. Matrix Anal. Appl. 14, 927 (1993). An implementation of the algorithm, by C. B. Mendl, was obtained from the MATLAB file exchange (file No. 46794). 